\title{
First Degree Family History Thyroid
}

\section{Cancer Positive}

National Cancer Institute

\section{Source}

National Cancer Institute. First Degree Family History Thyroid Cancer Positive. NCI

Thesaurus. Code C160211.

There is a first degree family history of thyroid cancer. 